\title{
COVID-19 (SARS-CoV-2 Infection) and Children: Pediatric Neurologist's Perspective
}

\author{
Prateek Kumar Panda ${ }^{1} \cdot$ Indar Kumar Sharawat ${ }^{1}$ (C)
}

Received: 5 April 2020 / Accepted: 27 April 2020 / Published online: 6 May 2020

(C) Dr. K C Chaudhuri Foundation 2020

To the Editor: The currently ongoing COVID-19 pandemic has involved more than 190 countries and children are affected less frequently. Although until now no specific isolated neurotropic symptoms of COVID-19 have not been reported in children, extrapolation from adult studies reveals a minority proportion of affected cases may develop neurological symptoms due to other organ dysfunction, dyselectrolytemia, hypoxia, adverse effects of drugs and only rarely due to direct invasion of the virus into the central nervous system [1]. Ischemic stroke, seizures, hypoxic encephalopathy, acute hemorrhagic encephalopathy and Guillain-Barré syndrome have been reported in adults [1-3].

International League Against Epilepsy (ILAE) and epilepsy foundation recommends that only Dravet syndrome affected children, children receiving steroids, Everolimus or other immunotherapies and those who have co-morbidities should be considered high risk for developing complications with COVID-19. Due to strict isolation measures in many countries, there is a high probability that children with epilepsy in developing countries might be deprived of continuing their antiepileptic regimen [4].

Even treatment regimens for neuromuscular diseases in children requiring hospital procedures including Nusinersen, Alglucosidase-Alfa, Intravenous immunoglobulin (IVIG) and Rituximab infusions for neuromuscular diseases are likely to be adversely impacted. Health care authorities should implement corrective measures to ensure the continuous availability of these life-saving medications by home visiting or at outreach centers. World Muscle Society recommends continuing steroids and other immunotherapies in children with Duchenne muscular dystrophy (DMD), myasthenia, inflammatory myopathy and other neuromuscular diseases during

Indar Kumar Sharawat

sherawatdrindar@gmail.com

1 Pediatric Neurology Division, Department of Pediatrics, All India Institute of Medical Sciences, Rishikesh, Uttarakhand 249203, India this pandemic. Steroids should not be stopped suddenly if the child gets COVID-19 and stress dose of steroids should be continued. Apart from the children on immunosuppressants, children with Forced vital capacity (FVC) $<60 \%$, cardiac involvement, severe kyphoscoliosis, weak cough, and airway clearance or those requiring home ventilation are at an increased risk and should adhere to strict isolation and other protective measures like hand washing and social distancing. Prone ventilation, often practiced in COVID-19 patients with Acute respiratory distress syndrome (ARDS), is difficult in children with cerebral palsy and advanced neuromuscular diseases.

Chloroquine and Azithromycin may affect neuromuscular junction transmission and should be used cautiously in children with myasthenia. Hydroxychloroquine associated neuropsychiatric effects have been already reported in adults suffering from COVID-19 and requires caution in adolescents too [5]. Rarely in children, Lopinavir/Ritonavir may also cause confusion or drowsiness. Adolescents in long term home isolation are at risk for developing depression, withdrawn behavior, and even suicidal tendency. Lastly, as many as $25 \%$ survivors of previous Middle East respiratory syndrome coronavirus (MERS-CoV) and Severe acute respiratory syndrome coronavirus (SARS-CoV) showed features of post-traumatic stress disorder and suffered from prolonged social stigma from peers and neighborhood due to widespread false rumors in the community. Thus, the COVID-19 survivors should be provided with adequate psychiatric advice before discharge.

\section{Compliance with Ethical Standards}

Conflict of Interest None.

\section{References}

1. Dong Y, Mo X, Hu Y, et al. Epidemiological characteristics of 2143 pediatric patients with 2019 coronavirus disease in China. Pediatrics. 2020. https://doi.org/10.1542/peds.2020-0702. 
2. Poyiadji N, Shahin G, Noujaim D, Stone M, Patel S, Griffith B. COVID-19-associated acute hemorrhagic necrotizing encephalopathy: CT and MRI features. Radiology. 2020;201187.

3. Zhao H, Shen D, Zhou H, Liu J, Chen S. Guillain-Barré syndrome associated with SARS-CoV-2 infection: causality or coincidence? Lancet Neurol. 2020. Available at: https://www.thelancet.com/ journals/laneur/article/PIIS1474-4422(20)30109-5. Accessed 5 Apr 2020.

4. Concerns About COVID-19 (Coronavirus) and Epilepsy. Epilepsy Found. Available at: https://www.epilepsy.com/article/2020/3/ concerns-about-covid-19-coronavirus-and-epilepsy. Accessed Apr 5, 2020.

5. Yazdany J, Kim AHJ. Use of hydroxychloroquine and chloroquine during the COVID-19 pandemic: what every clinician should know. Ann Intern Med. 2020. https://doi.org/10.7326/M20-1334.

Publisher's Note Springer Nature remains neutral with regard to jurisdictional claims in published maps and institutional affiliations. 